\title{
Sommaire de la Déclaration du CCNI sur la vaccination antigrippale pour la saison 2020-2021
}

\author{
Kelsey Young ${ }^{1}$, Ian Gemmill ${ }^{2,3}$, Robyn Harrison ${ }^{4,5}$ au nom du Comité consultatif national de \\ l'immunisation (CCNI)*
}

\section{Résumé}

Contexte : Les données probantes sur la vaccination antigrippale sont en constante évolution. Le Comité consultatif national de l'immunisation (CCNI) présente chaque année des recommandations sur l'utilisation des vaccins antigrippaux saisonniers à l'Agence de la santé publique du Canada.

Objectifs : Résumer les recommandations du CCNI sur l'utilisation des vaccins antigrippaux pour la saison 2020-2021 et mettre en exergue les recommandations nouvelles et actualisées.

Méthodes : 1) Pour mettre à jour la formulation de la recommandation sur la vaccination antigrippale des travailleurs de la santé, le $\mathrm{CCNI}$ a réévalué les données probantes du point de vue de l'éthique et de l'acceptabilité, en accord avec son mandat récemment élargi. 2) Pour établir des recommandations sur le recours au vaccin vivant atténué contre l'influenza (VAI) chez les personnes infectées par le VIH, le Groupe de travail sur l'influenza a élaboré une stratégie de recherche prédéfinie pour répertorier toutes les études admissibles, évaluer leur qualité, et résumer et analyser les résultats selon le processus fondé sur les données probantes du CCNI. Le CCNI a formulé de nouvelles recommandations fondées sur l'évaluation des données probantes.

Résultats : 1) Le CCNI continue de recommander que les travailleurs de la santé et les autres fournisseurs de soins qui interviennent en établissements et en milieux communautaires se fassent vacciner chaque année contre la grippe, et que ce groupe soit inclus parmi ceux pour lesquels le vaccin antigrippal est particulièrement recommandé. 2) Le CCNI a conclu que le VAl était immunogène chez les enfants ayant une infection stable au VIH. De ce fait, il recommande que le VVAl peut être une option chez les enfants de 2 à 17 ans ayant une infection stable au $\mathrm{VIH}$, recevant un traitement antirétroviral hautement actif et dont le système immunitaire fonctionne relativement bien.

Conclusion : Le CCNI continue de recommander qu'un vaccin antigrippal adapté à l'âge soit proposé chaque année à toute personne de six mois et plus qui ne présente aucune contreindication à l'administration du vaccin, en accordant une importance particulière aux groupes pour lesquels la vaccination antigrippale est particulièrement recommandée.
Cette oeuvre est mise à la disposition selon les termes de la licence internationale Creative Commons Attribution 4.0

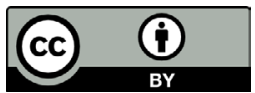

Affiliations

${ }^{1}$ Centre de l'immunisation et des maladies respiratoires infectieuses, Agence de la santé publique du Canada, Ottawa, ON

2 Président du Groupe de travail sur I'influenza du CC

${ }^{3}$ Université Queen, Kingston, ON

${ }^{4}$ Vice-président du Groupe de travail sur l'influenza du CCNI

${ }^{5}$ Université de l'Alberta; Services de santé Alberta, Edmonton, $A B$

\section{*Correspondance :}

phac.naci-ccni.aspc@canada.ca

Citation proposée : Young K, Gemmill I, Harrison R, au nom du Comité consultatif national de l'immunisation (CCNI). Sommaire de la Déclaration du CCNI sur la vaccination antigrippale pour la saison 2020-2021. Relevé des maladies transmissibles au Canada 2020;46(5):148-54. https://doi.org/10.14745/ccdr.v46i05a06f

Mots-clés : Comité consultatif national de l'immunisation, CCNI, influenza, grippe, vaccin antigrippal, conseils

\section{Introduction}

L'épidémie de grippe saisonnière est à l'origine d'une morbidité et d'une mortalité importantes dans la population canadienne (1) et pèse lourdement sur le système de santé lors de chaque saison grippale. Bien que l'épidémiologie de la grippe varie d'une année à l'autre, on estime que l'infection grippale cause en moyenne 12200 hospitalisations (2) et 3500 décès (3) chaque année.

Compte tenu de la nature cyclique de la grippe saisonnière, de la fréquence des changements dans les souches virales en 
circulation, et du nombre de vaccins antigrippaux autorisés au Canada, le Comité consultatif national de l'immunisation (CCNI) présente chaque année ses recommandations sur la vaccination antigrippale saisonnière à l'Agence de la santé publique du Canada (ASPC). Pour la saison grippale 2020-2021, le CCNI a mis à jour la formulation de sa recommandation sur la vaccination des travailleurs de la santé (TS) et à présenté une nouvelle recommandation sur le recours au vaccin vivant atténué contre I'influenza (VVAl) chez les personnes infectées par le VIH. Des renseignements complets sur le vaccin antigrippal se trouvent dans la Déclaration du CCNI sur la vaccination antigrippale pour la saison 2020-2021 (4) et dans les publications connexes. Cet article vise à fournir un résumé des renseignements contenus dans cette déclaration annuelle sur la grippe saisonnière et à mettre en exergue les mises à jour importantes.

\section{Abréviations des vaccins antigrippaux}

Les abréviations employées par le CCNI ont été récemment mises à jour pour mieux décrire les caractéristiques déterminantes des différents types de vaccins antigrippaux. Les abréviations actuelles sont énumérées au tableau 1.

\section{Tableau 1 : Abréviations des vaccins antigrippaux employées par le CCNI}

Catégorie

de vaccin

contre

I'influenza

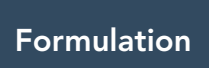

Type

Nouvelle

abréviation

du CCNIa

\begin{tabular}{|c|c|c|c|}
\hline \multirow{4}{*}{$\begin{array}{l}\text { Vaccin } \\
\text { inactivé } \\
\text { contre } \\
\text { l'influenza } \\
\text { (VII) }\end{array}$} & \multirow{3}{*}{ Trivalent (VII3) } & $\begin{array}{l}\text { À dose standard }{ }^{\mathrm{b}} \text {, } \\
\text { sans adjuvant, } \\
\text { administré par } \\
\text { voie IM }\end{array}$ & VII3-SD \\
\hline & & $\begin{array}{l}\text { Avec adjuvantc, } \\
\text { administré par } \\
\text { voie IM }\end{array}$ & VII3-Adj \\
\hline & & $\begin{array}{l}\text { À haute dosed, } \\
\text { sans adjuvant, } \\
\text { administré par } \\
\text { voie IM }\end{array}$ & VII3-HD \\
\hline & $\begin{array}{l}\text { Quadrivalent } \\
\text { (VII4) }\end{array}$ & $\begin{array}{l}\text { À dose standard }{ }^{\mathrm{b}} \text {, } \\
\text { sans adjuvant, } \\
\text { administré par } \\
\text { voie IM }\end{array}$ & VII4-SD \\
\hline $\begin{array}{l}\text { Vaccin vivant } \\
\text { atténué } \\
\text { contre } \\
\text { l'influenza } \\
\text { (WVAl) }\end{array}$ & $\begin{array}{l}\text { Quadrivalent } \\
\text { (VVAI4) }\end{array}$ & $\begin{array}{l}\text { Sans adjuvant, } \\
\text { Vaporisateur nasal }\end{array}$ & VAI4 \\
\hline
\end{tabular}

Abréviations : IM, intramusculaire; VII, vaccin inactivé contre l'influenza; VII3, vaccin trivalent inactivé contre l'influenza; VII3-Adj, vaccin trivalent inactivé contre I'influenza avec adjuvant; VII3-HD, vaccin trivalent inactivé contre l'influenza à haute dose; VII3-SD, vaccin trivalent inactivé contre I'influenza à dose standard; VII4, vaccin quadrivalent inactivé contre l'influenza; VII4-SD, vaccin quadrivalent inactivé contre l'influenza à dose standard; VAl, vaccin vivant atténué contre I'influenza; VVAI4, vaccin quadrivalent vivant atténué contre l'influenza

a Le suffixe numérique correspond au nombre d'antigènes contenus dans le vaccin (le chiffre « 3 " renvoie à la formulation trivalente et le chiffre « 4 », à la formulation quadrivalente). Le suffixe composé «-SD » renvoie aux produits VII sans adjuvant qui contiennent $15 \mu \mathrm{g}$ d'hémagglutinine (HA) par souche et qui sont administrés par voie intramusculaire en dose de 0,5 mL; «-Adj » renvoie à un VII avec adjuvant (p. ex. VII3-Adj pour FluadMD ou Fluad Pédiatrique ${ }^{\mathrm{MD}}$ ); «-HD » renvoie à un VII dont la quantité d'antigènes est supérieure à $15 \mu \mathrm{g}$ de $\mathrm{HA}$ par souche

(p. ex. VIII-HD pour Fluzone ${ }^{\mathrm{MD}}$ Haute dose)

b $15 \mu \mathrm{g}$ de HA par souche

c $7,5 \mu \mathrm{g}$ (dans $0,25 \mathrm{~mL}$ ) ou $15 \mu \mathrm{g}$ (dans $0,5 \mathrm{~mL}$ ) de HA par souche

$60 \mu \mathrm{g}$ de HA par souche

Source : Tableau tiré de la Déclaration du CCNI sur la vaccination antigrippale pour la saison 2020-2021 (4)

\section{Méthodes}

Pour préparer la Déclaration sur la vaccination antigrippale pour la saison 2020-2021, le Groupe de travail sur I'influenza a reconnu la nécessité de procéder à un examen des données probantes relatives à deux thématiques particulières et, après examen et analyse de l'information, a présenté des recommandations nouvelles ou actualisées selon le processus fondé sur les données probantes du CCNI (5). Le CCNI a soumis les données probantes existantes à une évaluation critique et validé les recommandations présentées.

\section{Vaccination des travailleurs de la santé et des autres fournisseurs de soins}

Le CCNI a reconnu la nécessité de réévaluer la formulation de la recommandation sur la vaccination antigrippale des TS et des autres fournisseurs de soins. Pour éclairer cette nouvelle formulation, les données probantes issues de quatre essais cliniques randomisés par grappes (6-9) destinés à évaluer I'incidence de la vaccination antigrippale des TS intervenant dans les établissements de soins gériatriques de longue durée ont été réévaluées et considérées du point de vue de l'éthique et de l'acceptabilité. L'éthique et l'acceptabilité ont été systématiquement prises en considération, selon les méthodes approuvées par le CCNI pour l'évaluation de l'éthique, de l'équité, de la faisabilité et de l'acceptabilité, et conformément au mandat récemment élargi du CCNI.

\section{Recours au vaccin vivant atténué contre l'influenza chez les personnes infectées par le $\mathrm{VIH}$}

Le Groupe de travail sur l'influenza du CCNI a encadré la réalisation d'une revue systématique visant à faciliter l'élaboration des lignes directrices sur le recours au WVAI chez les personnes infectées par le VIH. Des recherches ont été effectuées dans six bases de données électroniques (MEDLINE, EMBASE, Scopus, ProQuest Public Health Database, ClinicalTrials.gov et PROSPERO) depuis sa création et jusqu'au 13 avril 2018 pour répertorier la littérature pertinente sur l'efficacité potentielle, l'efficacité réelle, l'immunogénicité et l'innocuité du VVAl chez les adultes et les enfants de six mois et plus atteints du VIH. Des recherches ont également été effectuées dans le Système canadien de surveillance des effets secondaires suivant l'immunisation (SCSESSI) afin de répertorier les rapports faisant état aux effets secondaires après l'administration du WVI aux personnes infectées par le $\mathrm{VIH}$. Deux évaluateurs ont examiné de manière indépendante les titres et les résumés des articles recueillis dans le cadre de la recherche et les articles complets admissibles en vue de leur inclusion. Un évaluateur a extrait les données des études admissibles et évalué la qualité méthodologique de ces études selon les critères établis par Harris et coll. (10). Un autre évaluateur a validé l'extraction de données et l'évaluation de 
la qualité. Une synthèse narrative des données extraites a été réalisée.

\section{Résultats}

\section{Vaccination des travailleurs de la santé et des autres fournisseurs de soins}

S'appuyant sur sa réévaluation des données probantes du point de vue de l'éthique et de l'acceptabilité, le CCNI continue de recommander que, sauf contre-indications, les TS et les autres fournisseurs de soins qui interviennent en établissements et en milieux communautaires se fassent vacciner chaque année contre la grippe. Les TS et les autres fournisseurs de soins sont susceptibles de transmettre la grippe aux personnes à risque élevé et, du fait de leur activité et des contacts étroits avec les personnes à risque élevé de complications liées à la grippe, ils sont eux-mêmes exposés à un risque accru d'infection (11). Compte tenu du risque de transmission grippale et du risque accru d'infection, et sachant que la vaccination est le moyen le plus efficace de prévenir la grippe, le CCNI recommande l'inclusion de ce groupe parmi ceux pour lesquels l'administration du vaccin antigrippal est particulièrement recommandée. Le CCNI considère l'administration du vaccin antigrippal comme un volet essentiel de la norme de soins applicables à tous les TS et autres fournisseurs de soins pour protéger leur santé et celle de leurs patients. Ce groupe devrait considérer que la vaccination antigrippale annuelle relève de sa responsabilité à prodiguer des soins de la plus grande qualité.

De plus amples renseignements sur la recommandation du CCNI d'inclure les TS dans le groupe pour lesquels la vaccination antigrippale est particulièrement recommandée figurent à la section III. 2 de la Déclaration sur la vaccination antigrippale pour la saison 2020-2021 (4) du CCNI.

\section{Recours au vaccin vivant atténué contre l'influenza chez les personnes infectées par le VIH}

La revue systématique a permis de répertorier huit articles décrivant les résultats de cinq études visant à examiner l'immunogénicité et/ou l'innocuité de l'administration du WVAl chez les personnes infectées par le VIH. Aucune étude examinant l'efficacité potentielle ou réelle du WVAl chez cette population n'a été répertoriée. S'appuyant sur les données probantes recueillies, le $\mathrm{CCNI}$ a conclu que le VVAl était immunogène chez les enfants ayant une infection stable au VIH, recevant un traitement antirétroviral hautement actif (HAART) et dont le système immunitaire fonctionne relativement bien. Le CCNI a également conclu que, bien que le nombre de données probantes directes soit insuffisant pour détecter les effets secondaires rares associés au recours au WAI chez les enfants atteints du VIH, ce vaccin semblait présenter un profil d'innocuité similaire à celui du vaccin inactivé contre l'influenza (VII). Par ailleurs, il se peut que certains enfants (ou les personnes qui décident en leur nom) préfèrent que le vaccin antigrippal leur soit administré par vaporisation intranasale plutôt que par voie intramusculaire (IM), même si les préférences peuvent varier. Concernant le recours au WAI chez les adultes infectées par le $\mathrm{VIH}$, le $\mathrm{CCNI}$ a conclu que le nombre de données probantes disponibles sur l'immunogénicité et l'innocuité du vaccin chez les adultes infectées par le VIH était insuffisant pour justifier une recommandation sur le recours au WAl chez ce groupe d'âge. S'appuyant sur son évaluation des données probantes, le CCNI a présenté la recommandation suivante :

Le CCNI recommande que le VVAl peut être une option chez les enfants de 2 à 17 ans ayant une infection stable au VIH, recevant un HAART et dont le système immunitaire fonctionne relativement bien* (recommandation discrétionnaire du CCNI).

* Le VVAl doit seulement être envisagé chez les enfants infectées par le VIH qui répondent aux critères suivants :

- Ils reçoivent un HAART depuis au moins 4 mois

- Le nombre de leurs récepteurs CD4 est égal ou supérieur à $500 / \mu \mathrm{L}$ s'ils ont entre 2 et 5 ans ou à $200 / \mu \mathrm{L}$ s'ils ont entre 6 et 17 ans (nombre mesuré dans les 100 jours précédant l'administration du vaccin)

- Le taux d'acide ribonucléique (ARN) du VIH dans leur plasma est inférieur à 10000 copies/mL (nombre mesuré dans les 100 jours précédant l'administration du vaccin)

Bien que le CCNI et le Groupe canadien de recherche pédiatrique et périnatale sur le $\mathrm{VIH} / \mathrm{SIDA}$ considèrent toujours la vaccination antigrippale par voie IM comme la norme pour les enfants atteints du VIH, il serait raisonnable d'administrer le WAI aux enfants répondant aux critères ci-dessus si le sujet (ou la personne qui décide en son nom) refuse que le vaccin lui soit administré par voie IM.

Les résultats complets de cette revue et des renseignements complémentaires à l'appui de cette recommandation se trouvent dans la Déclaration du CCNI sur la recommandation du recours au vaccin vivant atténué contre l'influenza (WVI) chez les personnes infectées par le VIH (12).

\section{Résumé des recommandations du CCNI sur I'utilisation des vaccins antigrippaux pour la saison 2020-2021}

Le CCNI continue de recommander la vaccination antigrippale à toute personne âgée de six mois et plus qui ne présente aucune contre-indication à l'administration du vaccin. La vaccination devrait être proposée en priorité aux personnes qui présentent un risque élevé de complications ou d'hospitalisation liées à la grippe, aux personnes susceptibles de transmettre la grippe à des sujets à risque élevé de complications, et à d'autres personnes comme l'illustre le liste 1 . 


\section{Liste 1 : Groupes pour lesquels le vaccine antigrippal est particulièrement recommandé}

\section{Personnes présentant un risque élevé de complications ou d'hospitalisation liées à la grippe :}

- Toutes les femmes enceintes

- Adultes et enfants atteints d'une des affections chroniques suivantes ${ }^{a}$.

- Maladies cardiaques ou pulmonaires (notamment dysplasie bronchopulmonaire, fibrose kystique et asthme)

- Diabète sucré ou autres maladies métaboliques

- Cancer, troubles liés à l'immunodépression (résultant d'une maladie sous-jacente, d'un traitement, ou des deux, par exemple la greffe d'un organe plein ou de cellules souches hématopoïétiques)

- Néphropathie

- Anémie ou hémoglobinopathie

- Troubles neurologiques ou du développement neurologique (ces troubles comprennent les troubles neuromusculaires, neurovasculaires, neurodégénératifs et du développement neurologique ainsi que les troubles convulsifs [et, pour les enfants, les convulsions fébriles et les retards de développement isolés], mais ils excluent les migraines et les troubles psychiatriques sans troubles neurologiques)

- Obésité morbide (indice de masse corporelle [IMC] $\geq 40$ )

- Enfants et adolescents (de 6 mois à 18 ans) sous traitement pendant de longues périodes par de l'acide acétylsalicylique, en raison de la possibilité d'un risque accru de syndrome de Reye associé à la grippe

- Résidents de maisons de soins infirmiers et d'autres établissements de soins de longue durée, quel que soit leur âge

- Personnes de 65 ans et plus

- Tous les enfants de 6 à 59 mois

- Autochtones

Personnes qui pourraient transmettre la grippe à des sujets à risque élevé :

- Travailleurs de la santé et autres fournisseurs de soins dans des établissements et en milieux communautaires qui, par leurs activités, pourraient transmettre la grippe à des sujets à risque élevé de complications

- Contacts familiaux (adultes et enfants) de personnes à risque élevé de complications liées à la grippe, que ces dernières aient été vaccinées ou non :

- Contacts familiaux de personnes à risque élevé

- Contacts familiaux des nourrissons âgés de moins de 6 mois, qui sont à risque élevé de complications grippales, mais qui ne peuvent pas recevoir un vaccin contre la grippe

- Membres d'un ménage devant accueillir un nouveau-né durant la saison grippale

- Personnes qui s'occupent régulièrement d'enfants de 0 à 59 mois, que ce soit à la maison ou à l'extérieur

- Personnes qui fournissent des services à des sujets à risque élevé dans un milieu fermé ou relativement fermé (p. ex. équipage de navire)

\section{Autres :}

- Personnes qui fournissent des services communautaires essentiels

- Personnes en contact direct avec de la volaille infectée par le virus de la grippe aviaire durant les activités d'abattage

a Consulter Immunisation des personnes atteintes de maladies chroniques et Immunisation des sujets immunodéprimés dans la Partie 3 du GCl pour en savoir plus sur la vaccination des personnes atteintes de maladies chroniques (13)

Source : Tableau tiré de la Déclaration du CCNI sur la vaccination antigrippale pour la saison 2020-2021 (4)

Les recommandations concernant le choix du vaccin antigrippal par groupe d'âge et par posologie et voie d'administration selon l'âge sont résumées aux tableau 2 et tableau 3, respectivement.
Tableau 2 : Recommandations concernant le choix du vaccin antigrippal pour le processus décisionnel à l'échelle individuellea, par groupe d'âge

\begin{tabular}{|c|c|c|}
\hline $\begin{array}{l}\text { Groupe } \\
\text { d'âge }\end{array}$ & $\begin{array}{l}\text { Types de } \\
\text { vaccins } \\
\text { autorisés }\end{array}$ & $\begin{array}{c}\text { Recommandations concernant le choix du } \\
\text { vaccin antigrippal }\end{array}$ \\
\hline 6 à 23 mois & $\begin{array}{l}\text { - } \mathrm{VII3-SD} \\
\text { - } \mathrm{VII3-Adj} \\
\text { - } \mathrm{VII4-SD}\end{array}$ & $\begin{array}{l}\text { - Administrer le vaccin antigrippal } \\
\text { quadrivalent aux nourrissons pour qui il } \\
\text { n'est pas contre-indiqué, compte tenu du } \\
\text { fardeau du virus de la grippe B dans ce } \\
\text { groupe d'âge et de la possibilité de non- } \\
\text { concordance entre la souche prédominante } \\
\text { du virus de la grippe B en circulation et la } \\
\text { souche d'un vaccin trivalent } \\
\text { - En l'absence d'un vaccin quadrivalent, l'un } \\
\text { ou l'autre des vaccins trivalents doit être } \\
\text { utilisé }\end{array}$ \\
\hline 2 à 17 ans $^{b}$ & $\begin{array}{l}\text { - VII3-SD } \\
\text { - VII4-SD } \\
\text { - VVAI4 }\end{array}$ & 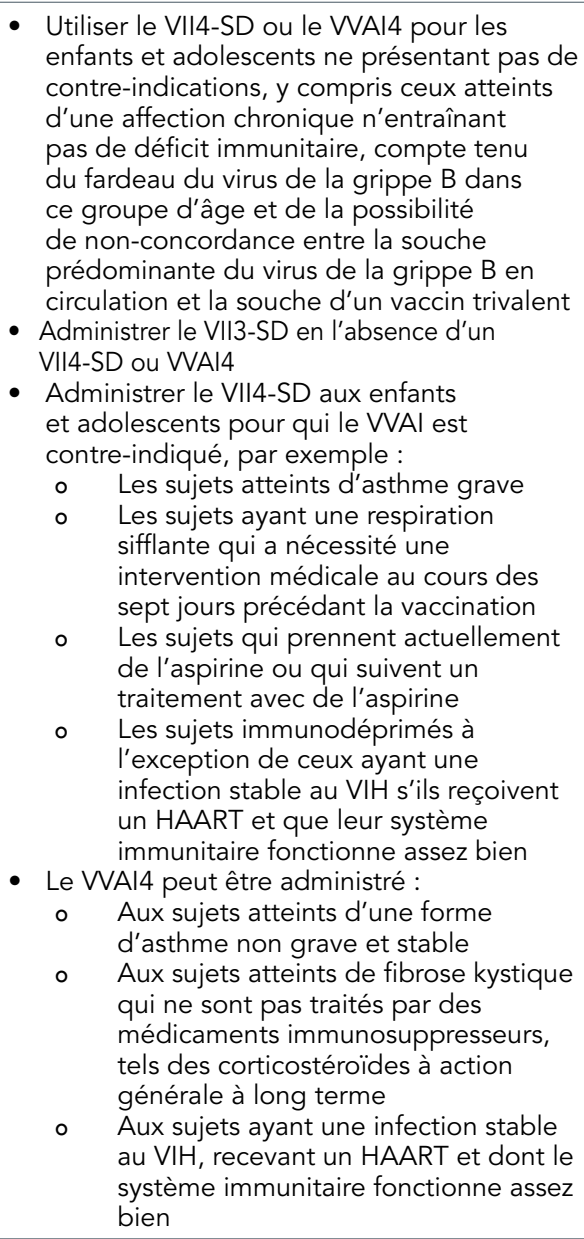 \\
\hline 18 à 59 ans & $\begin{array}{l}\text { - VII3-SD } \\
\text { - VII4-SD } \\
\text { - VVAI4 }\end{array}$ & $\begin{array}{l}\text { - Utiliser l'un ou l'autre des vaccins } \\
\text { antigrippaux disponibles chez les adultes ne } \\
\text { présentant aucune contre-indication } \\
\text { - Le VII doit être administré aux adultes } \\
\text { pour qui le WVAl est contre-indiqué ou non } \\
\text { recommandé, par exemple : } \\
\text { - Les femmes enceintes } \\
\text { - Les adultes atteints d'une affection } \\
\text { chronique figurant dans la tableau 2, y } \\
\text { compris un déficit immunitaire } \\
\text { - Les TS }\end{array}$ \\
\hline 60 à 64 ans & $\begin{array}{l}\text { - VII3-SD } \\
\text { - VII4-SD }\end{array}$ & $\begin{array}{l}\text { - Tous les vaccins antigrippaux disponibles } \\
\text { peuvent être utilisés chez ceux qui n'ont pas } \\
\text { de contre-indications }\end{array}$ \\
\hline
\end{tabular}


Tableau 2 : Recommandations concernant le choix du vaccin antigrippal pour le processus décisionnel à l'échelle individuellea, par groupe d'âge (suite)

\begin{tabular}{|c|c|c|}
\hline $\begin{array}{c}\text { Groupe } \\
\text { d'âge }\end{array}$ & $\begin{array}{l}\text { Types de } \\
\text { vaccins } \\
\text { autorisés }\end{array}$ & $\begin{array}{l}\text { Recommandations concernant le } \\
\text { choix du vaccin antigrippal }\end{array}$ \\
\hline 65 ans et plus ${ }^{c}$ & $\begin{array}{ll}\text { - } & \text { VIII-SD } \\
\text { - VII3-Adj } \\
\text { - VII3-HD } \\
\text { VIIIS-SD }\end{array}$ & $\begin{array}{l}\text { - Le VII3-HD devrait être utilisé plutôt que } \\
\text { le VII3-SD, compte tenu du fardeau de la } \\
\text { maladie associée à la grippe A(H3N2) et } \\
\text { des données probantes suffisantes à l'appui } \\
\text { d'une meilleure protection du VII3-HD par } \\
\text { rapport au VII3-SD chez les adultes de } 65 \text { ans } \\
\text { et plus } \\
\text { ○ Le CCNI ne formule pas des } \\
\text { recommandations comparatives à } \\
\text { l'échelle individuelle sur I'utilisation du } \\
\text { VIII-Adj ou du VII4-SD plutôt que du } \\
\text { VIII-SD ou entre le VII3-Adj, le VIII-HD } \\
\text { et le VII4-SD } \\
\text { Â défaut d'un de ces produits, } \\
\text { administrer un des vaccins } \\
\text { antigrippaux disponibles }\end{array}$ \\
\hline
\end{tabular}

Abréviations : HAART, traitement antirétroviral hautement actif; TS, travailleur de la santé; $\mathrm{VII}$, vaccin inactivé contre l'influenza; VII3-Adj, vaccin trivalent inactivé contre l'influenza avec adjuvant; VII3-HD, vaccin trivalent inactivé contre l'influenza à haute dose; VII3-SD, vaccin trivalent inactivé contre l'influenza à dose standard; VII4-SD, vaccin quadrivalent inactivé contre l'influenza à dose standard; VVAl, vaccin vivant atténué contre l'influenza; WAI4, vaccin quadrivalent vivant atténué contre l'influenza

Les recommandations relatives au processus décisionnel à l'échelle individuelle sont destinées aux personnes souhaitant se protéger contre la grippe, ou aux vaccinateurs souhaitant conseiller certains patients sur la prévention de la grippe

b Se reporter au tableau 4 de la Déclaration du CCNI sur la vaccination antigrippale pour la saison 2020-2021 pour obtenir un résumé des caractéristiques du VVAI par rapport au VII chez les saison 2020-2021 pour

enfants de 2 à 17 ans (4)

"Se reporter au tableau 5 de la Déclaration du CCNI sur la vaccination antigrippale pour la saison 2020-2021 pour obtenir une comparaison des caractéristiques des différents types de vaccins antigrippaux proposés aux adultes de 65 ans et plus (4)

Source : Tableau adapté de la Déclaration du CCNI sur la vaccination antigrippale pour la saison 2020-2021 (4)

\section{Tableau 3 : Posologie et voie d'administration recommandées, selon l'âge, pour les types de vaccins antigrippaux offerts pour la saison 2019-2020}

\begin{tabular}{|c|c|c|c|c|c|}
\hline \multirow[b]{2}{*}{ Groupe d'âge } & \multicolumn{4}{|c|}{$\begin{array}{c}\text { Type de vaccin antigrippal } \\
\text { (voie d'administration) }\end{array}$} & \multirow[b]{2}{*}{$\begin{array}{l}\text { Nombre de doses } \\
\text { requises }\end{array}$} \\
\hline & $\begin{array}{c}\text { VII3-SDa ou } \\
\text { VII4-SD } \\
\text { (Intramusculaire) }\end{array}$ & $\begin{array}{c}\text { VII3-Adjc } \\
\text { (Intramusculaire) }\end{array}$ & $\begin{array}{c}\text { VII3-HD } \\
\text { (Intramusculaire) }\end{array}$ & $\begin{array}{c}\text { VVAle } \\
\text { (Intranasal) }\end{array}$ & \\
\hline 6 à 23 mois & $0,5 \mathrm{~mL}^{f}$ & $0,25 \mathrm{~mL}$ & - & - & 1 ou $2^{g}$ \\
\hline 2 à 8 ans & $0,5 \mathrm{~mL}$ & - & - & $0,2 \mathrm{~mL}(0,1 \mathrm{~mL}$ par narine $)$ & 1 ou $2^{9}$ \\
\hline 9 à 17 ans & $0,5 \mathrm{~mL}$ & - & - & $0,2 \mathrm{~mL}(0,1 \mathrm{~mL}$ par narine $)$ & 1 \\
\hline 18 à 59 ans & $0,5 \mathrm{~mL}$ & - & - & $0,2 \mathrm{~mL}(0,1 \mathrm{~mL}$ par narine $)$ & 1 \\
\hline 60 à 64 ans & $0,5 \mathrm{~mL}$ & - & - & - & 1 \\
\hline 65 ans et plus & $0,5 \mathrm{~mL}$ & $0,5 \mathrm{~mL}$ & $0,5 \mathrm{~mL}$ & - & 1 \\
\hline
\end{tabular}

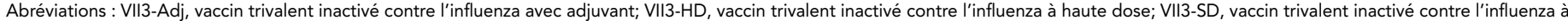
dose standard; VII4-SD, vaccin quadrivalent inactivé contre l'influenza à dose standard; VAI4, vaccin quadrivalent vivant atténué contre l'influenza

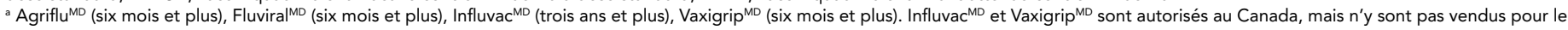
moment

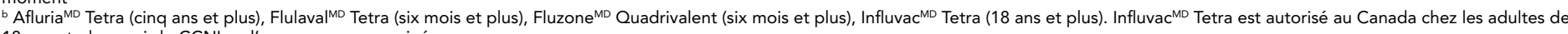
18 ans et plus, mais le CCNI ne l'a pas encore examiné

c Fluad Pédiatrique ${ }^{\mathrm{MD}}$ ( 6 à 23 mois) ou Fluad ${ }^{\mathrm{MD}}$ (65 ans et plus)

${ }^{d}$ Fluzone ${ }^{\mathrm{MD}}$ Haute Dose ( 65 ans et plus)

e FluMist ${ }^{\mathrm{MD}}$ Quadrivalent (2 à 59 ans)

${ }^{\dagger}$ Des données semblent indiquer une amélioration modérée de la réponse immunitaire chez les nourrissons, sans qu'il y ait augmentation de la réactogénicité, après l'administration de doses

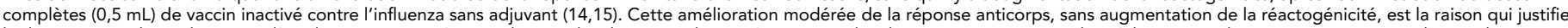
l'administration d'une dose complète du vaccin inactivé sans adjuvant chez les sujets de tous âges. Pour plus de renseignements, voir la Déclaration sur la vaccination antigrippale pour la saison 2011-2012 (16)

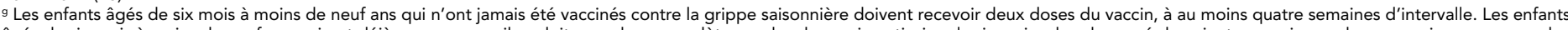

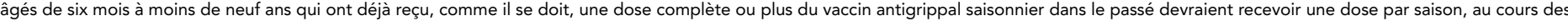
années suivantes

Source : Tableau tiré de la Déclaration du CCNI sur la vaccination antigrippale pour la saison 2020-2021 (4) 


\section{Déclaration des auteurs}

K. Y. - Rédaction de la version préliminaire, révision et édition I. G. - Révision et édition

R. H. - Révision et édition

Le document Chapitre sur la grippe du Guide canadien

d'immunisation et Déclaration du Comité consultatif national de l'immunisation (CCNI) sur la vaccination antigrippale pour la saison 2020-2021 a été préparé par K. Young, A. Sinilaite, L. Zhao et I. Gemmill au nom du Groupe de travail sur l'influenza du CCNI et a été approuvé par le CCNI.

\section{Conflit d'intérêts}

Aucun.

\section{Remerciements}

Membres du Groupe de travail sur I'influenza : I. Gemmill (président), R. Harrison (vice-présidente), C. Bancej,

L. Cochrane, N. Dayneka, L. Grohskopf, D. Kumar, J. Langley,

P. Wolfe-Roberge, J. McElhaney, A. McGeer, D. Moore, S. Smith, B. Warshawsky et J. Xiong

Membres du CCNI : C. Quach (présidente), S. Deeks

(vice-présidente), N. Dayneka, P. De Wals, V. Dubey, R. Harrison, K. Hildebrand, C. Rotstein, M. Salvadori, B. Sander, N. Sicard et S. Smith

Représentants de liaison : L. M. Bucci (Association canadienne de santé publique), E. Castillo (Société des obstétriciens et gynécologues du Canada), A. Cohn (Centers for Disease Control and Prevention, États-Unis), J. Emili (Collège des médecins de famille du Canada), M. Naus (Comité canadien sur I'immunisation), D. Moore (Société canadienne de pédiatrie) et A. Pham-Huy (Association pour la microbiologie médicale et I'infectiologie Canada)

Anciens représentants d'office : J. Gallivan (Direction des produits de santé commercialisés, Santé Canada [SC]), E. Henry (Centre de l'immunisation et des maladies respiratoires infectieuses [CIMRI]), Agence de la santé publique du Canada [ASPC]), M. Lacroix (Groupe consultatif en matière d'éthique en santé publique, ASPC), J. Pennock (CIMRI, ASPC), R. Pless (Direction des produits biologiques et des thérapies génétiques, SC), G. Poliquin (Laboratoire national de microbiologie, ASPC) et T. Wong (Direction générale de la santé des Premières nations et des Inuits, Services aux Autochtones Canada)

Le Comité consultatif national de l'immunisation remercie vivement les personnes suivantes de leur contribution à cette déclaration : O. Baclic, A. House, S. Ismail, M. Laplante et M. Tunis.

\section{Financement}

Les travaux du Comité consultatif national de l'immunisation bénéficient du soutien de l'Agence de la santé publique du Canada.

\section{Références}

1. Statistique Canada. Les dix principales causes de décès, 2011. Ottawa (ON) : Statistique Canada; 2018. https:// www150.statcan.gc.ca/n1/pub/82-625-x/2014001/ article/11896-fra.htm

2. Schanzer DL, McGeer A, Morris K. Statistical estimates of respiratory admissions attributable to seasonal and pandemic influenza for Canada. Influenza Other Respir Viruses 2013 Sep;7(5):799-808. DOl PubMed

3. Schanzer DL, Sevenhuysen C, Winchester B, Mersereau T. Estimating influenza deaths in Canada, 1992-2009. PLoS One 2013 Nov;8(11):e80481. DOI PubMed

4. Comité consultatif national de l'immunisation. Chapitre sur la grippe du Guide canadien d'immunisation et Déclaration sur la vaccination antigrippale pour la saison 2020-2021. Ottawa (ON) : ASPC; 2020. https://www.canada.ca/fr/ sante-publique/services/publications/vaccins-immunisation/ guide-canadien-immunisation-declaration-vaccinationantigrippale-2020-2021.html

5. Comité consultatif national de l'immunisation. Recommandations pour l'immunisation fondées sur des données probantes - Méthodes du Comité consultatif national de l'immunisation. Relevé des maladies transmissibles au Canada 2009 Jan;35(ACS-1):1-10. https:// www.canada.ca/fr/sante-publique/services/rapportspublications/releve-maladies-transmissibles-canada-rmtc/ numero-mensuel/2009-35/methodes-comite-consultatifnational-immunisation.html

6. Carman WF, Elder AG, Wallace LA, McAulay K, Walker A, Murray GD, Stott DJ. Effects of influenza vaccination of health-care workers on mortality of elderly people in long-term care: a randomised controlled trial. Lancet 2000 Jan;355(9198):93-7. DOI PubMed

7. Hayward AC, Harling R, Wetten S, Johnson AM, Munro S, Smedley J, Murad S, Watson JM. Effectiveness of an influenza vaccine programme for care home staff to prevent death, morbidity, and health service use among residents: cluster randomised controlled trial. BMJ 2006 Dec;333(7581):1241. DOI PubMed

8. Potter J, Stott DJ, Roberts MA, Elder AG, O'Donnell B, Knight PV, Carman WF. Influenza vaccination of health care workers in long-term-care hospitals reduces the mortality of elderly patients. J Infect Dis 1997 Jan;175(1):1-6. DOI PubMed

9. Lemaitre $M$, Meret $T$, Rothan-Tondeur $M$, Belmin J, Lejonc JL, Luquel L, Piette F, Salom M, Verny M, Vetel JM, Veyssier P, Carrat F. Effect of influenza vaccination of nursing home staff on mortality of residents: a cluster-randomized trial. J Am Geriatr Soc 2009 Sep;57(9):1580-6. DOI PubMed

10. Harris RP, Helfand M, Woolf $\mathrm{SH}$, Lohr KN, Mulrow CD, Teutsch SM, Atkins D; Methods Work Group, Third US Preventive Services Task Force. Current methods of the US Preventive Services Task Force: a review of the process. Am J Prev Med 2001 Apr;20(3 Suppl):21-35. DOI PubMed 
11. Kuster SP, Shah PS, Coleman BL, Lam PP, Tong A, Wormsbecker A, McGeer A. Incidence of influenza in healthy adults and healthcare workers: a systematic review and meta-analysis. PLoS One 2011;6(10):e26239. DOI PubMed

12. National Advisory Committee on Immunization. $\mathrm{NACl}$ Recommendation on the use of live-attenuated influenza vaccine (LAIV) in HIV-infected individuals. Ottawa (ON): Public Health Agency of Canada; 2018.

13. Agence santé publique du Canada. Guide canadien d'immunisation : Partie 3 - Vaccination de populations particulières. Ottawa (ON) : ASPC; 2015. https://www. canada.ca/fr/sante-publique/services/publications/ vie-saine/guide-canadien-immunisation-partie3-vaccination-populations-particulieres/page-7-immun isation-personnes-atteintes-maladies-chroniques.html
14. Langley JM, Vanderkooi OG, Garfield HA, Hebert J, Chandrasekaran V, Jain VK, Fries L. Immunogenicity and safety of 2 dose levels of a thimerorsal-free trivalent seasonal influenza vaccine in children aged 6-35 months: A randomized, controlled trial. J Pediatric Infect Dis Soc 2012 Mar;1(1):55-63. DOI PubMed

15. Skowronski DM, Hottes TS, Chong M, De Serres G, Scheifele DW, Ward BJ, Halperin SA, Janjua NZ, Chan T, Sabaiduc S, Petric M. Randomized controlled trial of dose response to influenza vaccine in children aged 6 to 23 months. Pediatrics 2011 Aug;128(2):e276-89. DOl PubMed

16. Comité consultatif national de l'immunisation. Déclaration sur la vaccination antigrippale pour la saison 2011-2012. Relevé des maladies transmissibles au Canada 2011;37(ACS5):1-61. https://www.canada.ca/content/dam/phac-aspc/ migration/phac-aspc/publicat/ccdr-rmtc/11vol37/acs-dcc-5/ assets/pdf/acs-dc««-5-fra.pdf 